\title{
Research on the Key Technology and Method of Indoor Localization Based on WLAN
}

\author{
Zhe Chen, Yanxiang He, and Wenxue Yang
}

\begin{abstract}
With the widespread use of wireless networks and development of pervasive computing technology, location based services (LBS) have attracted more and more attention and shown great energy in many applications, such as emergency, moving target tracking and customized information delivery. Localization technology is a prerequisite and key to for LBS to be implemented. Common Localization methods include TOA (Time of Arrival) and AOA (Angle of Arrival). In this paper proposed TOA\&AOA composite positioning method combines both of their features, reduces the required positioning wireless APs (Access Points), simplifies the location environment, improves the efficiency of locating. This paper through theoretical analysis and simulation comparison proves that the composite positioning accuracy is within acceptable range.
\end{abstract}

Index Terms-Location based services (LBS), wireless local area network (WLAN), time of arrival (TOA), angle of arrival (AOA), measurement error

\section{INTRODUCTION}

Localization technology has begun to walk into people's ordinary life, with the optimization of network environment as well as the popularity of mobile data service. More and more applications have been developed with localization technology. Hence, it is very important to improve the localization accuracy and applicability.

As we all know, GPS (Global Localization System) technology has been quite mature, which is used by positioning and navigation for moving objects such as ships, automobiles, aircraft and pedestrians. But the technology has poor localization accuracy and there exists some limitations for the indoor environment [1].

As the indoor positioning accuracy and other requirements, the current indoor wireless positioning technology research focuses mainly on the WLAN (Wireless Local Area Network), RFID (Radio Frequency Identification), Bluetooth, infrared, ZigBee, UWB (Ultra-wide Band) etc., these methods have both advantages and disadvantages. Some positioning range is limited, such as infrared positioning technology, some system stability is poor, easy to be disturbed, such as Bluetooth positioning technology. Also some method itself has many advantages, but is not easily integrated into other systems that require separate physical device support [2]. At present the research of WLAN indoor

Manuscript received May 9, 2013; revised July 25, 2013

F. A. Zhe Chen is with the Computer School, Wuhan University and School of Computer Science, China University of Geosciences, Wuhan, Hubei, P. R. China (e-mail: wdchenzhe@163.com).

S. B. Yanxiang He is with Computer School, Wuhan University, Wuhan, Hubei, P. R. China (e-mail: yxhe@whu.edu.cn).

T. C. Wenxue Yang is with the School of Electron and Information, Hubei Three Gorges Polytechnic, Yichang, Hubei, P. R. China (e-mail: yangwx@xinhuanet.com). localization technology is a hot topic.

\section{CONCEPT}

\section{A. WLAN Localization Technology}

WLAN Positioning technology using WiFi terminals (such as a laptop, PDA or smart phone, etc.) scans parameters which are defined in the WLAN protocols, receives signal strength or signal-to-noise ratio information from multiple wireless APs. Due to the fact that the current WLAN has widely covered office buildings, universities, airports and other places, WLAN positioning technology can make full use of the existing resources. As WLAN-based indoor positioning technology takes full advantage of the existing WLAN resources, received signal strength defined in the 802.11 protocol as acquisition signal, as well as a higher position accuracy fingerprinting positioning [3]. Therefore, based on the WLAN the indoor positioning system technology has comprehensive advantages, such as low-cost, high positioning accuracy. It has received extensive attention from many domestic and foreign scholars and research institutions and commercial corporations.

\section{B. Indoor Localization Method Based on WLAN}

Indoor navigation system requires a good positioning method, positioning method refers to how the navigation system employs the received measurement information to calculate the measured object position. In the positioning method, TOA and AOA method have many advantages, such as intuitive principles, simple arrangement and easy implementation, suitable for running on an intelligent wireless terminal in navigation system [4]. But the TOA method requires more measurement points, AOA method positioning performance declines with the positioning space increased. This paper makes the combination of the two characteristics, makes full use of their advantages, forming a composite TOA \& AOA positioning method, further reducing required numbers for positioning APs, simplifies the positioning environment. While taking advantages of the TOA and AOA method, improving the positioning efficiency and simulation error analysis proves that complex positioning accuracy in positioning methods can meet the needs of indoor navigation [5].

\section{METHOD}

In the traditional TOA based on the indoor WLAN positioning system, the measuring units are some fixed location APs, and the measured object and APs transfer the position measurement information over the wireless. These measurements information includes signal propagation time 
information between the AP and measured object. Wireless electromagnetic signal propagates in the air, the propagation speed is equal to the speed of light, the propagation time can be used to measure the distance between the measured object and the AP. Position of the measured object can be calculated by the known location of the APs [6]. In fact, different positioning system has a number of different positioning methods, such as TOA, AOA, etc., these two kinds of typical locating method are described below.

\section{A. TOA Method}

TOA positioning technology measures the propagation time from the AP electromagnetic signal to the measured object, calculates the distance from the AP to the measured object by propagation time, and then calculates position coordinates using the geometric triangle relationship as shown in Fig. 1 [7].

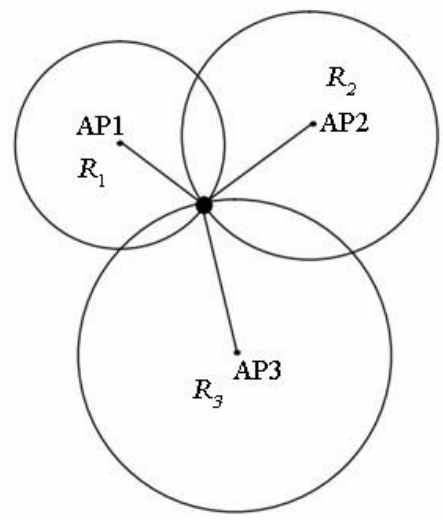

Fig. 1. Chart of TOA.

Once the distance $R i$ from the object to the $A P$ is obtained, then the target position must be located at the circumference of a circle with radius $R_{i}$. The measured object coordinates $\left(x_{0}\right.$, $\left.y_{0}\right)$ and the coordinate $\left(x_{i}, y_{i}\right)$ of the $A P_{i}$ meet the following formulas:

$$
\left\{\begin{array}{l}
\left(x_{1}-x_{0}\right)^{2}+\left(y_{1}-y_{0}\right)^{2}=r_{1}^{2} \\
\left(x_{2}-x_{0}\right)^{2}+\left(y_{2}-y_{0}\right)^{2}=r_{2}^{2} \\
\left(x_{3}-x_{0}\right)^{2}+\left(y_{3}-y_{0}\right)^{2}=r_{3}^{2}
\end{array}\right\}
$$

By solving the equations (1), you can get the measured object position $\left(x_{0}, y_{0}\right)$. Therefore, the TOA positioning algorithm, also known as peripheral positioning method, or three circles intersection positioning method.

As for the TOA, the time synchronization accuracy and time measurement errors have a great bearing on positioning accuracy. When positioning, the measured object needs to know the signal propagation time, and the measured object and APs have a very precise clock, so the implementation is quite difficult [8]. Therefore, using TOA positioning has a certain difficulty in the indoor environment.

\section{B. AOA Method}

AOA is a positioning method which determines the measured object position using the angle of the arrival wireless signal as shown in Fig. 2, the measured object position can be represented by the angles between the measured object and a group of APs. The following is a two-dimensional horizontal space, the measured target position calculation equations are given as:

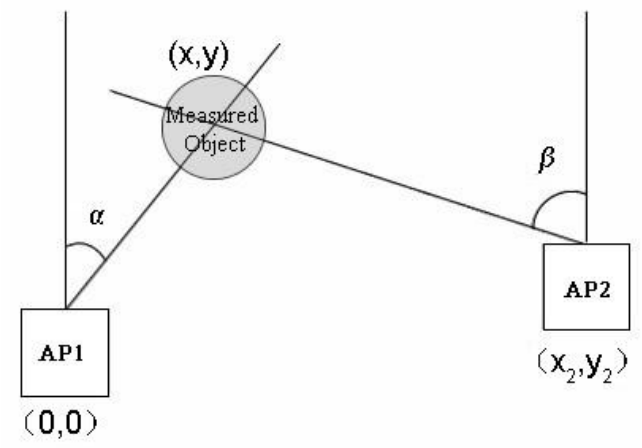

Fig. 2. Chart of AOA.

Assuming that two AP coordinates are known, respectively $(0,0)$ and $\left(x_{2}, y_{2}\right)$. The unknown measured object coordinates of $(x, y)$ can be obtained from the figure above.

$$
\begin{aligned}
& \frac{x}{y}=\tan \alpha \\
& \frac{x_{2}-x}{y-y_{2}}=\tan \beta
\end{aligned}
$$

The AOA method is simple and straightforward in terms of calculation principle, and easy to implement positioning algorithm, however, the disadvantage is encountered when in large space, its measure error grows faster than in the TOA method [9].

\section{Composite Positioning Method (TOA \& AOA)}

TOA and AOA methods are the use of the arrival time and the arrival angle calculation respectively to obtain the measured object location, it is natural to take into account whether we can simultaneously use these two measurements? Since TOA and AOA method has its own advantage and proper scope, this paper presents a TOA \& AOA composite positioning method. This positioning method employs the angles and distances between the measured object and the measured $A P s$ and calculates the measured object spatial coordinates [10]. From the point of view of the number of measuring units, composite positioning method requires less measurement units than the TOA or AOA methods. In TOA method, if you want to measure the two-dimensional coordinates, it must be based on at least three $A P$. If you want to measure the three-dimensional coordinates of the detected objects, positioning systems require more APs (at least four). In AOA method, in order to determine the two-dimensional coordinates of the measured object requires only two $A P$, however, as three-dimensional positioning takes four $A P S$. TOA \& AOA composite method requires only two AP and can provide three-dimensional position of the measured object, because in some practical applications the number of APs must be limited, to minimize the demand for the APs. This method will greatly enhance the actual maneuverability, more conducive to the deployment and control.

In a three dimensional space, the two $A P$ is placed at a fixed known location. The three-dimensional coordinates of the APs are known parameters [11]. Symbol d is known fixed distance between the two $A P, h$ is the $A P$ height from the ground. TOA method measures the distances $\mathrm{d}_{1}$ and $\mathrm{d}_{2}$ of the 
$A P 1$ and $A P 2$ to the measured objects respectively. $\alpha$ is the angle lying in between plane $\mathrm{a}_{1}$ and plane $b_{1}$. Similarly, $\beta$ is the angle between plane $a_{2}$ and plane $b_{2}$.

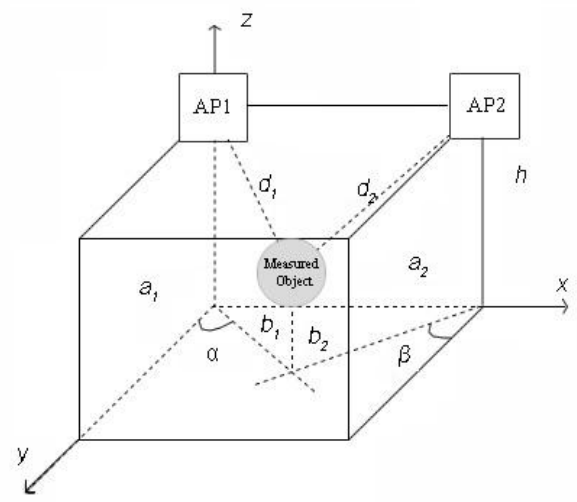

Fig. 3. Chart of TOA \& AOA.

The distances and angles are all obtained and the measured object position can be determined, as shown in Fig. 3. The measured object coordinate $(x, y, z), d_{1}, d_{2}, \alpha, \beta$ meet the following equations:

$$
\begin{gathered}
(h-z)^{2}+(x / \sin \alpha)^{2}=d_{1}^{2} \\
(h-z)^{2}+(x / \sin \beta)^{2}=d_{2}^{2} \\
x+y \tan \beta=d
\end{gathered}
$$

From the Fig. 3

$$
\begin{aligned}
& x / y=\tan \alpha \\
& \frac{d-x}{y}=\tan \beta
\end{aligned}
$$

From equation (7) and (8)

$$
\begin{aligned}
& \frac{d-x}{\frac{x}{\tan \alpha}}=\tan \beta \\
& \frac{y-y \tan \alpha}{y}=\tan \beta
\end{aligned}
$$

The measured object can obtain coordinates $x, y$ by equation (9) and (10) calculation formula. The measured object height $\mathrm{z}$ can be achieved by equation (11):

$$
z=h-\sqrt{d_{2}^{2}-\frac{y^{2}}{\cos ^{2} \beta}}
$$

\section{Positioning ERror ANALYSIS METHOD}

According to the above-mentioned introduction, the TOA method, if you want to measure the two-dimensional coordinates of the measured object, must be based on at least three APs. If you want to measure the three-dimensional coordinates of the measured objects, positioning systems require more APs (at least four). In the AOA method, only two APs can determine the two-dimensional coordinates of the measured object, three-dimensional positioning requires four APs [12].

TOA \& AOA composite method requires only two AP and can provide three-dimensional position of the measured object. So it is easy to deploy and control. On one hand this method requires fewer units of measurement than the TOA method or the AOA method, on the other hand it easily obtains high precision than two methods above, detailed analysis is as follows.

Through the composite positioning method, we can obtain the position coordinates, and whether this position is accurate, whether measure error is acceptable, so we need to verify the problems in practical application, and this error needs to conduct effectiveness analysis. Although the AOA method principle is very simple, but in practicable applications, there are some limitations, it is assumed that the mobile terminal can measure the angle of the transmitted signal to reach the AP. Its location depends entirely on the measured angle accuracy, if the mobile terminal and the AP is not far away from each other, there is little environmental disturbance, the AOA positioning method works well. In fact the AP angular resolution is restricted, once the environment was severely disturbed, the measurement angle error will increase meanwhile. The TOA method determines the distance by measuring the propagation time, the measuring time error is independent of the space, only on the device timer precision of the measurement space. Measuring angle error is related to the size of space and the complexity of environment, so in large space, the AOA accuracy is lower than TOA method [13].

\section{A. Estimate the TOA Positioning Error}

In the case of three-dimensional positioning, the $A P$ coordinate $\left(x_{i}, y_{i}\right)$ is known ahead of time, and the direct measurement result depends on the arrival time $t$, assuming that the measurement time error is $\Delta t$, then $d=c \times t$, can be draw $\Delta d=c \times \Delta t$.

According equation (2) and error propagation formulas to estimate the positioning error is given as:

$$
\begin{aligned}
\Delta x & =\left(d_{2}+d_{1}\right) \times \Delta d \\
& =\left(d_{2}+d_{1}\right) \times c \times \Delta t \\
& =c^{2} \times\left(t_{2}+t_{1}\right) \times \Delta t \\
\Delta y & =c^{2} \times\left(t_{3}+t_{1}\right) \times \Delta t \\
\Delta z & =c^{2} \times\left(t_{4}+t_{1}\right) \times \Delta t
\end{aligned}
$$

While the arrival time $\mathrm{t}$ and the measurement time error $\Delta t$ values are small, however, due to the amplification effect of the light speed $-c^{2}$, and from the foregoing analysis we can see that the positioning error is large.

\section{B. Estimate the TOA\&AOA Composite Positioning Error}

From the equation (9) and (10), the following equations can be achieved,

$$
x=\frac{d \times \tan \alpha}{\tan \alpha+\tan \beta}
$$




$$
y=\frac{d}{\tan \alpha+\tan \beta}
$$

where $d$ is the fixed distance between the two $A P$ and is a known quantity. $\alpha$ and $\beta$ is the measured angle independent of each other, assuming error $\Delta \alpha$ and $\Delta \beta$

$$
\begin{gathered}
\Delta x=\frac{\Delta \alpha}{\tan ^{3} \alpha}+\frac{\frac{\Delta \alpha}{\tan ^{2} \alpha}+\frac{\Delta \beta}{\tan ^{2} \beta}}{\tan \alpha+\tan \beta} \\
\Delta y=d \times \frac{\frac{\Delta \alpha}{\tan ^{2} \alpha}+\frac{\Delta \beta}{\tan ^{2} \beta}}{\tan \alpha+\tan \beta}
\end{gathered}
$$

According to the equation (11)

$$
\Delta z=\frac{d_{1} \times c \Delta t+\frac{x}{\sin \alpha} \times\left(\frac{\Delta x}{\sin \alpha}+\frac{|\cos \alpha| \Delta \alpha}{\sin ^{2} \alpha}\right)}{\sqrt{d_{1}^{2}-\frac{x^{2}}{\sin ^{2} \alpha}}}
$$

\section{RESULT}

The following takes the three-dimensional positioning for example, through MATLAB simulates respectively TOA method and the composite method. Simulation process sets the different spatial range (assuming cubic space), through analysizing two positioning method simulation results and the actual spatial positions, to compare their performance quality [14]. In TOA method, the assumed measurement time error is $\Delta t=10^{-10}$ between the measured object and the $A P$, and the propagation times $t 1, t 2, t 3$ is determined by simulation system randomly within a predetermined space, so the simulation results have universality. In the composite method, assuming the measurement time error $\Delta t=10^{-10}$, and assuming that the angle measurement error $\Delta \alpha=\Delta \beta=0.001$, in order to ensure result comparability at different spaces in the measurements range, let $\alpha=25^{\circ}, \beta=70^{\circ}$, the simulation results is shown in Table I.

TABLE I: THE COMPARISON OF POSITION ERROR OF TOA\&AOA IN DIFFERENT RANGES

\begin{tabular}{cccccc}
\hline \hline Position $(\mathrm{m})$ & 10 & 20 & 30 & 40 & 50 \\
\hline TOA & 1.0427 & 1.5429 & 1.8508 & 2.8869 & 3.5589 \\
TOA\&AOA & 0.4645 & 0.7694 & 1.1287 & 1.4929 & 1.5366 \\
Position $(\mathrm{m})$ & 60 & 70 & 80 & 90 & 100 \\
\hline TOA & 3.9912 & 4.1785 & 4.3784 & 4.6957 & 5.2134 \\
TOA\&AOA & 2.1438 & 2.2731 & 2.9623 & 3.2307 & 3.6993 \\
\hline \hline
\end{tabular}

The simulation results and comparisons can be further illustrated figuratively as shown in Fig. 4.

From the above results, the composite positioning method has an acceptable error range. When the space $10 \mathrm{~m} \times 10 \mathrm{~m} \times 10 \mathrm{~m}$ cube, the composite error of positioning is $0.4645 \mathrm{~m}$, relative to the spatial distance, the error representing small value [15]. From the Table I and Fig. 4 above we can come to the conclusion that the result error in the composite method is smaller, and positioning is higher accuracy than in than TOA positioning method, and with the spatial expansion of the scope, the positioning accuracy of the composite method has improved greatly compared with TOA [16].

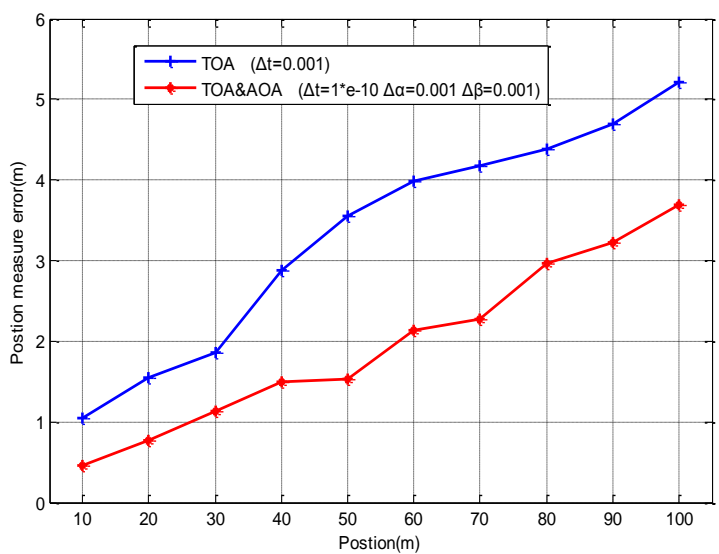

Fig. 4. Comparison of position error of TOA\&AOA in different ranges

Through the error analysis, we can see that the composite positioning method for indoor navigation system with $10 \mathrm{~m}$ of the smallest unit is already full enough. Through this positioning method to determine the location coordinates have the error control in a controllable range, fully capable of indoor navigation needs.

\section{CONCLUSION}

This article first resolves the indoor positioning method selection problem. It puts forward to combines the TOA method and the AOA method together, using the TOA \& AOA composite positioning method. This Method reduces AP number of the positioning needed, simplify the positioning process while ensuring the positioning accuracy and other performance indicators, and carry on simulation error analysis. It is proved that the composite positioning method has certain advantages in indoor accuracy positioning performance.

\section{ACKNOWLEDGMENT}

F. A. Zhe Chen thanks to his wife, Huaqin Zeng, for her constant support and unwavering confidence in him. This work would not have been possible without the meticulous guidance and encouragement of Zhe Chen's adviser, Dr. Yanxiang He.

\section{REFERENCES}

[1] V. Lang and C. Gu, "A locating method for WLAN based location service," in Proc. IEEE International Conference on e-Business Engineering, 2005, pp. 427-431.

[2] Y. Y. Gu and A. Lo, "A survey of indoor localization systems for wireless personal networks," in Proc. IEEE Transactions on Signal Processing, pp. 13-32, Nov. 2009.

[3] A. P. Jardosh, K. Papagiannaki, and E. M. Belding, "Green WLANs: on-demand WLAN infrastructures," Mobile Networks and Applications, pp. 798-814, Dec. 2009

[4] T. P. Deasy and W. G. Scanlon, "Algorithms for improving the accuracy of both deterministic and probabilistic methods in WLAN-based indoor user localization," International Journal Of Wireless Information Networks, pp. 207-216, Oct. 2004

[5] H. Khoury and V. Kamat, "Evaluation of position tracking technologies for user localization in indoor construction environments," Automation In Construction, pp. 444-457, July 2009 
[6] D. Carevic, "Automatic estimation of multiple target positions and velocities using passive TDOA measurements of transients," in Proc. IEEE Transactions on Signal Processing, pp. 424-436, Feb. 2007

[7] X. Wang, S. Yuan, R. Laur, and W. Lang, "Dynamic localization based on spatial reasoning with RSSI in wireless sensor networks for transport logistics," Sensors and Actuators A: Physical, pp. 421-428, Nov. 2011

[8] L. Mengual, O. Marban, and S. Eibe, "Clustering-based location in wireless networks," Expert Systems with Applications, pp. 7552-7561, 2010

[9] A. W. Tsui, Y. H. Chuang, and H. H. Chu, "Unsupervised learning for solving RSS hardware variance problem in WiFi localization," Mobile Networks and Applications, pp. 677-691, 2009

[10] K. Curran, E. Furey, and T. Lunney, "An evaluation of indoor location determination technologies," Journal of Location Based Services, pp. $61-74,2011$

[11] F. Gustafsson and F. Gunnarsson, "Mobile positioning using wireless networks: Possibilities and fundamental limitations based on available wireless network measurements," in Proc. IEEE Signal Processing Magazine, pp. 41-53, 2007

[12] M. Hazas and A. Hopper, "Broadband ultrasonic location systems for improved indoor positioning," in Proc. IEEE Transactions Mobile Computing, pp. 536-547, 2006

[13] A. Ward, A. Jones, and A. Hopper, "A new location technique for the active office," Personal Communications, pp. 42-47, 2004

[14] K. Kamol, "Distribution of WLAN received signal strength indication for indoor location determination," in Proc. International Symposium on Wireless Pervasive Computing, pp. 6-11, 2006

[15] V. Honkavirta, T. Perala, and S. A. Loytty, "A comparative survey of WLAN location fingerprinting methods," in Proc. International Conference on Positioning, pp. 243-251, 2009

[16] M. Roshanei and M. Maleki, "Dynamic-KNN: A novel locating method in WLAN based on angle of arrival," in Proc. IEEE Symposium on Industrial Electronics and Applications, pp. 722-726, 2009

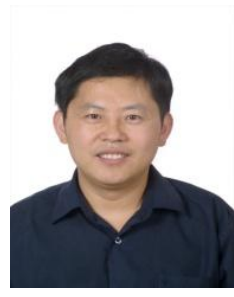

Zhe Chen was born in Wanjia Village, Wanggang Town of Xishui County, Huanggang city, Hubei province, China on October 12, 1973. Zhe Chen received his Bachelor's degree of Engineering in Computer and Applied Technology from Huazhong University of Science and Technology, Wuhan, China in 1995 and the Master degree of Engineering in Applied Technology of Computer from Wuhan University, Wuhan, China in 2003, and now are pursuing a PHD degree in Computer Software and Theory at Wuhan University, Wuhan, China under the guidance of Dr Yanxiang He. Zhe Chen was a programmer from the ground up and later a software engineer from 1996 through to 2000 in Hubei province textile co. LTD. Since 2003, he has been with the faculty of networking Engineering, school of Computer Science, China University of Geosciences in Wuhan where he is currently an assistant professor.

Zhe Chen has broad research interests ranging from Wireless Communications and Networking, Sensor Networks, Internet of Things, Delay/Disruption-Tolerant Networking, Software Engineering, High Performance Computing, and Big Data Processing.

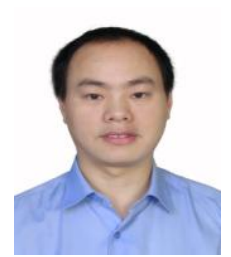

Wenxue Yang was born in Hanchuan city, Hubei, China in 1974. He received his Bachelor degree of Computer Software from Department of Computer and Mathematics, Hubei University, Wuhan, China in 1997, and the Master degree of Computer Software and Theory from the School of Computer Science, Chongqing Normal University, Chongqing, China in 2011. He was a programmer from the ground up and later an software engineer from 1997 through to 2011, currently he is working as an assistant professor in School of Electron and Information, Hubei Three Gorges Polytechnic, Yichang, Hubei, China. His area of interests includes Intelligent Computing, Data Mining and wireless Sensor networks. 